IBIMA Publishing

Advances in Stem Cells

http://www.ibimapublishing.com/journals/ASC/asc.html

Vol. 2013 (2013), Article ID 208514, 15 pages

DOI: $10.5171 / 2013.208514$

Research Article

\title{
Hela Cells Consist of Two Cell Types, as Evidenced by Cytochemical Staining for Alkaline Phosphatase Activity: A Possible Model for Cancer Stem Cell Study
}

\author{
Masahiro Sato ${ }^{1}$, Naoko Kubota ${ }^{2}$, Emi Inada ${ }^{2}$, Issei Saitoh ${ }^{3}$, Masato Ohtsuka ${ }^{4}$, \\ Shingo Nakamura ${ }^{5}$, Takayuki Sakurai ${ }^{6}$ and Satoshi Watanabe ${ }^{7}$ \\ ${ }^{1}$ Section of Gene Expression Regulation, Frontier Science Research Center, Kagoshima University, Japan \\ ${ }^{2}$ Department of Pediatric Dentistry, Graduate School of Medical and Dental Sciences, Kagoshima \\ University, Japan \\ ${ }^{3}$ Division of Pediatric Dentistry, Graduate School of Medical and Dental Science, Niigata University, \\ Chuo-ku, Japan \\ ${ }^{4}$ Division of Basic Molecular Science and Molecular Medicine, School of Medicine, Tokai University, \\ Isehara, Japan \\ ${ }^{5}$ Department of Surgery II, National Defense Medical College, Japan \\ ${ }^{6}$ Department of Organ Regeneration, Graduate School of Medicine, Shinshu University, \\ Matsumoto, Japan \\ ${ }^{7}$ Animal Genome Research Unit, Division of Animal Science, National Institute of Agrobiological Sciences, \\ Tsukuba, Japan
}

Received 24 November 2012; Accepted 7 January 2012; Published 27 February 2013

Academic Editor: Gérard Lizard

\section{Abstract}

Alkaline phosphatase (ALP) is known to be expressed in the several somatic stem cells and cancer cells. To investigate whether ALP may be a promising marker for cancer stem cells (CSCs), we examined the expression of ALP in human squamous cell carcinoma HeLa cells using a cytochemical

Copyright (C) 2013 Masahiro Sato, Naoko Kubota, Emi Inada, Issei Saitoh, Masato Ohtsuka, Shingo Nakamura, Takayuki Sakurai and Satoshi Watanabe. This is an open access article distributed under the Creative Commons Attribution License unported 3.0, which permits unrestricted use, distribution, and reproduction in any medium, provided that original work is properly cited. Contact author: Masahiro Sato E-mail: masasato@ms.kagoshima-u.ac.jp

How to Cite this Article: Masahiro Sato, Naoko Kubota, Emi Inada, Issei Saitoh, Masato Ohtsuka, Shingo Nakamura, Takayuki Sakurai and Satoshi Watanabe, "Hela Cells Consist of Two Cell Types, as Evidenced by Cytochemical Staining for Alkaline Phosphatase Activity: A Possible Model for Cancer Stem Cell Study," Advances in Stem Cells, vol. 2013, Article ID 208514, 15 pages

DOI: $10.5171 / 208514.767448$ 
staining kit. We found that approximately $40 \%$ of HeLa cells were positive for ALP activity. A single cell-derived colony assay revealed that the newly formed colonies could be classified into uniformly (U, 23\%), mosaically (M, 17\%), and non-stained (N, 60\%) colonies. Each colony was picked and cultured for 2 additional weeks for cell propagation; the cells were either M- (45\%) or N-type (55\%), suggesting that the U-type colonies may have spontaneously changed to M-type colonies during cultivation. These resulting $\mathrm{M}$ - or $\mathrm{N}$-type cells were stable with respect to ALP activity. DNA microarray analysis revealed that the gene expression pattern of N-type cells is almost identical to that of their parental HeLa cells (comprising M-type cells), but several genes including ALP gene were upregulated in the HeLa cells. Cultivation of single HeLa cell-derived colonies in the presence of the small molecule 6-bromoindirubin-3'-oxime (BIO), a potent inhibitor of glycogen synthase kinase 3 (GSK3), caused a reduction in the ratio of M-type colonies, suggesting that the transition from $\mathrm{U}$ - to M-type colonies is regulated by the Wnt/ $\beta$-catenin signaling pathway. Although there is no evidence, at present, that the ALP-positive cells are CSCs, future investigation may reveal that HeLa cells may be a good model for CSC study.

Keywords: Cancer stem cell; alkaline phosphatase; mosaic expression; HeLa cells.

\section{Introduction}

Recent data have demonstrated that tumors contain a small subpopulation of cells, namely, cancer stem cells (CSCs), which exhibit a capacity for self-renewal and which are responsible for tumor maintenance and metastasis (Lapidot et al., 1994; Bonnet and Dick, 1997; Singh et al., 2004; Al-Hajj and Clarke, 2004; Jordan et al., 2006). Although many exceptions have been reported, cellsurface molecules such as CD133 (prominin1), CD44 (hyaluronan receptor), and CD138 (syndecan-1) are generally considered markers for CSCs (Visvader and Lindeman, 2008). However, these molecules are expressed in the later stages of embryonic cells, such as blastocysts (a stage just prior to implantation), post-implanted early fetuses, and hematopoietic progenitor populations. For example, CD133/prominin-1 is expressed in trophoblasts, but not in cells of the inner cell mass (ICM) at the blastocyst stage (Kania et al., 2005). CD44 molecules are present in abundance in the liver of postimplantation fetuses at E (embryonic day) 10.5. Furthermore, as early as E 9.5, CD44positive cells can be detected in circulating blood (Wheatley et al., 1993). In the developing mouse, CD138/syndecan-1 is first detected throughout the embryo at the 4-cell stage (Sutherland et al., 1991).
Alkaline phosphatase (ALP) is an enzyme that hydrolyzes a broad range of monophosphate esters at alkaline pH optima. In humans, there are at least 4 distinct forms of APL: placental, intestinal, germ cell, and liver/bone/kidney (L/B/K) ALP (Mornet et al., 2001). The latter, $\mathrm{L} / \mathrm{B} / \mathrm{K}$ ALP, is expressed in numerous adult tissues, and therefore, is also called tissue non-specific ALP (TNAP). Aside from its expression in adult tissues, ALP is abundantly expressed in undifferentiated cells, such as ES cells, preimplantation embryos [2-cell embryos to blastocysts (ICM)] and embryonic ectoderm at the egg-cylinder stage, PGCs, and immature spermatogenic cells (Lepire and Ziomek, 1989; Hahnel et al., 1990; Ginsburg et al., 1990). It is also a marker of neuronal progenitor cells (Langer et al., 2007), human myogenic progenitor cells (also called 'pericytes') (Dellavalle et al., 2007), human dental pulp cells (Tomlinson et al., 2011), and mesenchymal stem cells (MSCs) in bone marrow (Battula et al., 2009; Sobiesiak et al., 2009). Given the cell/tissue localization pattern of ALP, it is likely that ALP can serve as a CSC marker, if tumor cells express ALP.

In a preliminary test, we examined expression of ALP in several established tumor cell lines using a cytochemical staining kit for ALP activity and found that some cell 
lines, including HeLa cells, exhibited mosaic expression of ALP (Fig. 1). Among them, HeLa cells were classified into ALP-positive [hereafter referred to as ALP (+)] and negative [hereafter referred to as ALP (-)] cells, with a ratio of roughly 2:3 (Fig. 1). Interestingly, these ALP (+) cells formed clusters in a single cell layer, suggesting clonal expansion of these cells (Fig. 1A). The mode of expansion of these ALP ( + ) cells in the HeLa cell line provoked us to suppose that the ALP (+) cells may be CSCs, from which ALP (-) cells have been derived.

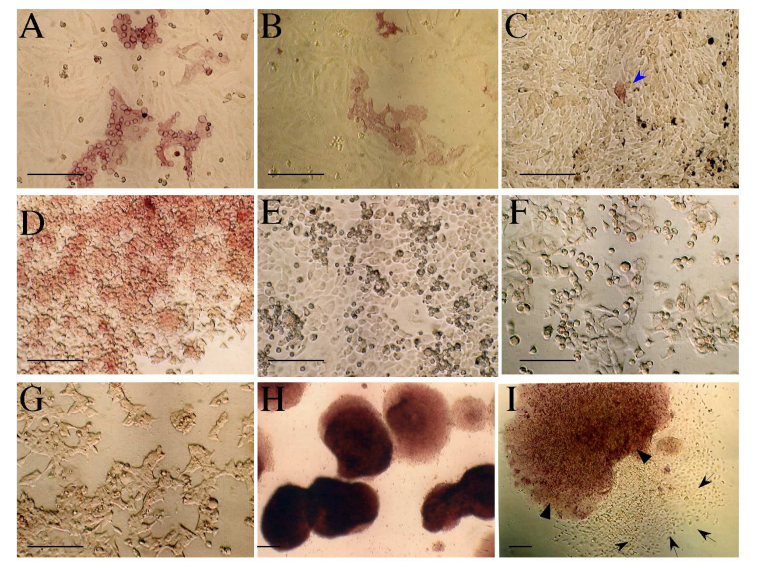

Figure 1. Cytochemical staining of several cultured cell lines for ALP activity. HeLa (A, B), B16 melanoma (C), PC12 (D), SUIT-2 (E), KP-2 (F), HEK-293 (G), and F9 (H) cell lines together with differentiated F9 cells (I) were subjected to cytochemical staining using an ALP detection kit. HeLa cells shown in (B) were obtained from Dr. Masayuki Ozawa (Kagoshima University Graduate School of Medical and Dental Sciences). Distinct ALP activity was observed in HeLa, PC12, and F

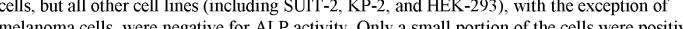
ALP activity [arrow in (C)] When F9 cells were induced to differentiate by application of $10^{-8} \mathrm{M}$ ALP activity [arrow in (C). When F9 cells were induced to differentiate by application of $10^{-8} \mathrm{M}$ F9 cells usually located at the central portion of a colony [arrowheads in (D)] were observed Bar = F9 cells 100 m.

In this study, we employed a traditional cell cloning method, namely, seeding of cells at a clonal density, to assess the interrelationship between ALP (+) and (-) cells in the HeLa cell line. In addition, we picked these colonies to test whether they are stable with respect to ALP activity during cultivation. Finally, these isolated clones were subjected to DNA microarray analysis to examine whether these two types of cells differ in their gene expression profiles.

\section{Materials and Methods}

\section{Cell Lines and Culture}

HeLa (CRL-1469 ${ }^{\mathrm{TM}}$ ), mouse pheomyeloma cell line PC12 (CRL-1721 ${ }^{\mathrm{TM}}$ ), mouse melanoma cell line B16 (CRL-6475 ${ }^{\mathrm{TM}}$ ), and HEK-293 (CRL-1573 ${ }^{\text {TM }}$ ) cells were purchased from the American Type Culture Collection (ATCC) (Manassas, VA). The human pancreatic cell line KP-2 (RCB1973) was obtained from the RIKEN BioResource Center Cell Bank (Tsukuba, Ibaraki, Japan). SUIT-2 is a cell line isolated from human pancreatic carcinoma (Iwamura et al., 1987). The nullipotent embryonal carcinoma cell line F9 (Bernstine et al., 1973) was used as a control for demonstrating that expression of ALP is decreased when these cells differentiate into endodermal cells. All these cells were grown in high glucose-DMEM (\#11995; Invitrogen Co., Carlsbad, CA) with $10 \%$ fetal bovine serum (FBS) and 100U/mL penicillin/streptomycin at $37^{\circ} \mathrm{C}$ in a humidified atmosphere of $5 \% \mathrm{CO}_{2}$ in air.

\section{Cell Cloning Assay}

HeLa cells after trypsinization were plated at a density of 3-10 cells/well (24-well plate; Iwaki Glass Ltd., Tokyo, Japan) and cultured 
for 7 days, by which time colonies comprising 20-40 cells appear. Some of these colonies were fixed with 4\% paraformaldehyde (PFA) in phosphatebuffered saline without $\mathrm{Ca}^{2+}$ and $\mathrm{Mg}^{2+}$ at $\mathrm{pH}$ 7.4 [PBS(-)] for $5 \mathrm{~min}$ at room temperature before being subjected to cytochemical staining for ALP activity, as described below. The other colonies were picked using a tryspin/EDTA-dipped small paper disc (3 MM Whatman paper, $3 \mathrm{~mm}$ in width, and 3 $\mathrm{mm}$ in length) to isolate pure cell populations, as described previously (Nakayama et al., 2007). These papers containing cells were then dipped into medium in wells of a 48-well plate (Iwaki Glass Ltd.) for propagation of each cell clone.

\section{Cytochemistry}

The ALP assay was performed using the Leukocyte Alkaline Phosphatase kit (SigmaAldrich Co., St. Louis, MO) following the manufacturer's instructions. Colonies stained uniformly and distinctly were designated as U-type colonies, as shown in Fig. 2E. These colonies consist only of ALP (+) cells. Similarly, colonies stained mosaically, which contain ALP (+) cells and one or more ALP (-) cells [very weakly (pale) or non-stained] were designated as M-type colonies, as shown in Fig. 2C, D. Colonies non-stained for ALP activity were designated as $\mathrm{N}$-type colonies, as shown in Fig. 2F.
A

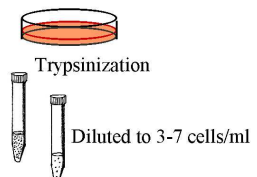

Plating

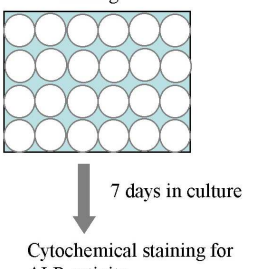

ALP activity

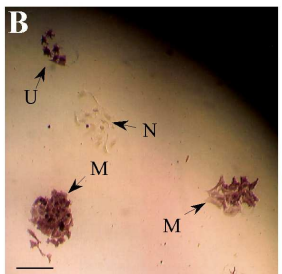

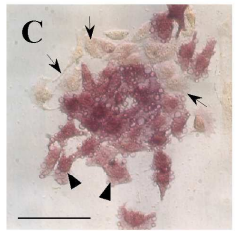
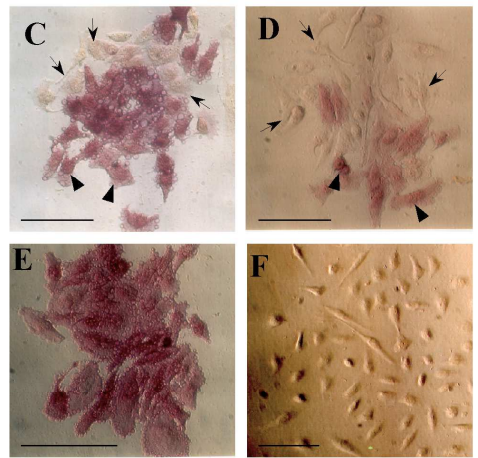

G

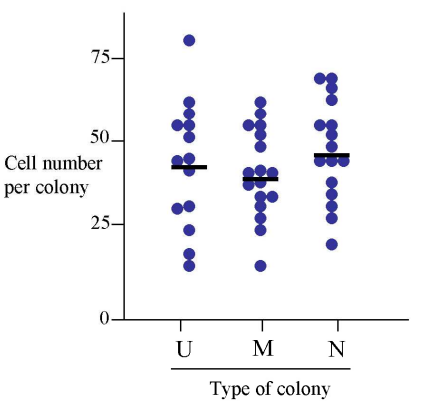

Figure 2. (A) Experimental flow for a single cell-derived colony assay. HeLa cells were plated at a clonal density into the wells of a 24-well plate. Seven days later the emerging colonies were fixed and subjected to cytochemical staining for ALP activity using an ALP staining kit. (B) ALP staining. Several colonies that had been stained uniformly (indicated by ' $U$ '), mosaically (indicated by ' $M$ '), or non-stained (indicated by ' $\mathrm{N}$ ') were observed in a well. Bar $=100 \mu \mathrm{m}$. (C-F) Classification of colonies. U-type colonies are designated as those comprised only of stained cells $(\mathbf{E})$. M-type colonies are a mixture of stained (indicated by arrows in $[\mathbf{C}, \mathbf{D}]$ ) and unstained (indicated by arrowheads in $[\mathbf{C}, \mathbf{D}]$ ) cells. Colonies having more than one pale or non-stained cell were classified as M-type colonies. N-type colonies, shown in (F), are designated as those comprised only of pale or unstained cells. (G) Counting the number of cells in each colony 7 days after seeding single HeLa cells into the wells of a 24-well plate. These colonies were fixed and then subjected to cytochemical staining for ALP activity for their classification. The cell number in each classified colony was recorded and plotted. Each dot corresponds to the cell number of a single
colony. 


\section{Cell Counting}

Colonies (7 days after seeding) derived from a single HeLa cell were fixed and then subjected to cytochemical staining for ALP activity. The number of cells in a colony was recorded by counting the cells under a light microscopy manually. More than 14 colonies of each type (U, M, and N) were recorded. Colonies with fewer than 10 cells were ignored, because they may have been composed of inadequate clones that are unable to proliferate properly.

\section{Treatment with 6-Bromoindirubin-3'- Oxime (BIO), a Potent Inhibitor of Glycogen Synthase Kinase 3 (GSK3)}

HeLa cells were plated in the wells of a 24well plate, as described in the Cell cloning assay section. Seven days after seeding, the medium was replaced by medium containing 1 or $3 \mu \mathrm{M}$ of BIO (GSK-3 Inhibitor IX; Calbiochem Inc., San Diego, CA) and cultured for 3 days. These cells were fixed with $4 \%$ PFA for $5 \mathrm{~min}$ at room temperature for cytochemical staining for ALP activity.

\section{RT-PCR}

Total RNA was isolated using an ISOGEN kit (Wako Pure Chemicals Inc., Tokyo, Japan).Four micrograms of total RNA from

the cell lines was reverse transcribed into cDNA using SuperScript $\AA$ II Reverse Transcriptase (Invitrogen Co.) and Oligo(dT) 20 primer (\#FSK-201; Toyobo Co. Ltd., Osaka, Japan) in a total of volume of 20 $\mu \mathrm{L}$. The cDNAs were amplified from undiluted cDNA samples $(1 \mu \mathrm{L})$ by 40 cycles of $45 \mathrm{~s}$ denaturation at $94^{\circ} \mathrm{C}, 45 \mathrm{~s}$ annealing at $58^{\circ} \mathrm{C}$, and $60 \mathrm{~s}$ extension at $72^{\circ} \mathrm{C}$, in a PC708 thermal cycler (Astec, Fukuoka, Japan). A negative, no-template control (designated as -RT) was included for each reaction. Information concerning the PCR primers used is listed in Table 1 . The resulting RT-PCR products were subjected to electrophoresis in 2\% agarose gels and visualized after staining with ethidium bromide.

\section{Hoechst Dye-Exclusion Assay}

HeLa cells were plated in the wells of a 24well plate, as described in Section 2.2. Seven days after seeding, colonies were incubated with $5 \mu \mathrm{g} / \mathrm{ml}$ Hoechst 33342 dye (Calbiochem Inc.) dissolved in high glucose$\mathrm{DMEM}+10 \% \mathrm{FBS}$ for $15 \mathrm{~min}$ at $37^{\circ} \mathrm{C}$, rinsed in PBS(-), and then fixed with $4 \%$ PFA for 5 min at room temperature. The fixed cells were next subjected to cytochemical staining for ALP activity, as described above. Fluorescent cells were visualized using an Olympus BX10 microscope with epifluorescent filters for Hoechst, and microphotographed using a digital camera (FUJIX HC-300/OL; Fuji Film, Tokyo, Japan) attached to the fluorescence microscope and printed using a Mitsubishi digital color printer (CP700DSA; Mitsubishi, Tokyo, Japan).

\section{DNA Microarray Analysis}

Total RNA was extracted using an SV Total RNA Isolation System (Promega) according to the manufacturer's instructions. RNA samples were quantified using an ND-1000 spectrophotometer (NanoDrop Technologies, Wilmington, DE) and the quality was confirmed with a Experion System (Bio-Rad Laboratories, Hercules, CA). The cRNA was amplified, labeled using a GeneChip $®$ WT Terminal Labeling and Control Kit, and hybridized to an Affymetrix Human Genome U133 Plus 2.0 array, according to the manufacturer's instructions. All hybridized microarrays were scanned by an Affymetrix scanner. Relative hybridization intensities and background hybridization values were calculated using Affymetrix Expression Console $^{\mathrm{TM}}$. Raw signal intensities for each probe were calculated from hybridization intensities. The raw signal intensities of two samples were $\log _{2}$-transformed and normalized by RMA and a quantile algorithm (Bolstad et al., 2003) with Affymetrix $®$ Expression Console ${ }^{\mathrm{TM}} 1.1$ software. To identify up- or downregulated genes, we calculated Z-scores (Quackenbush, 2002) and ratios (non-log scaled fold-change) from the 
normalized signal intensities of each probe for comparison between control and experiment sample. Then we established criteria for regulated genes: (upregulated genes) Z-score $\geq 2.0$ and ratio $\geq 1.5$-fold, (downregulated genes) Z-score $\leq-2.0$ and ratio $\leq 0.66$. The experimental data have been submitted to GEO (http://www.ncbi.nlm.nih.gov/geo/) under series accession number GSE42222.

\section{Results}

\section{Cell Cloning Assay}

As mentioned previously, HeLa cells are comprised of two cell types, namely ALP (-) and $(+)$ cells (Fig. 1A). To characterize these two types of cells, we first performed a cell cloning assay, as schematically shown in Fig. 2A. Seven days after seeding of single HeLa cells at a density of 3-7 cells/well in a 24well plate, the emerging colonies were cytochemically examined for ALP activity. These colonies were uniformly (U) stained, mosaically (M) stained, or non-stained (N) (Fig. 2B). Detailed analyses of these stained colonies demonstrated that of the 48 colonies examined, 11 (23\%), 8 (17\%), and 29 (60\%) were classified as $\mathrm{U}, \mathrm{M}$, and $\mathrm{N}$ types of colonies, respectively. In U-type colonies, all composite cells were ALP (+) (Fig. 2E), whereas in M-type colonies there was a mixture of ALP (+) and (-) cells, in which the ALP activity was greatly reduced (arrows in Fig. 2C, D). Interestingly, these ALP (-) cells tended to localize at a position surrounding the ALP (+) cells (arrows vs. arrowheads in Fig. 2C, D), suggesting that ALP (-) cells are derivatives of ALP (+) cells. The N-type colonies were completely negative for ALP activity (Fig. 2F).

The proliferation rates of U-, M-, and N-type colonies were assessed by counting cells in a colony generated 7 days after plating single HeLa cells onto a 24-well plate. There was no noticeable difference between the colony types in cell number per colony (Fig. 2G).

Next, using another plate into which single HeLa cells had been plated, the clonally expanded colonies were randomly picked using trypsin/EDTA-dipped small papers. The papers containing the cells were transferred to the wells of a 48-well plate. Seven days later, the growing cells in the wells were fixed and subjected to cytochemical staining for ALP activity (Fig. $3 \mathrm{~A})$. Of the 16 wells examined, 6 wells contained only ALP (-) cells (Fig. 3C), and the other 10 wells contained a mixture of ALP (+) and (-) cells (Fig. 3B). Concomitantly, cells in the remaining wells were trypsinized, replated to wells of a new 48-well plate and cultured up to confluency (about 3 weeks after colony isolation) (Fig. 3A). When the confluent cells were fixed and then subjected to cytochemical staining for ALP activity, 6 of the 12 wells examined contained a mixture of ALP (+) and (-) cells (Fig. 3D), and the other 6 wells contained ALP (-) cells alone (Fig. 3E). Of these ALP ( - ) cells, one clone, called ' $\mathrm{H}-1$,' was propagated and used for several assays as a control for HeLa cells [a mixture of ALP $(+)$ and ( - ) cells]. These results suggest that ALP (+) cells in U-type colonies may have converted to ALP (-) cells during the relatively long periods of culture, and once converted to ALP (-) cells, could not revert to ALP (+) cells, so long as they are maintained under normal conditions.

Based on these findings, we summarized the behavior of ALP (+) and (-) HeLa cells (Fig. 3F). Namely, ALP (+) cells exhibit selfrenewal, and also generate ALP (-) cells. ALP $(-)$ cells can proliferate as do the ALP (+) cells, but cannot revert to ALP (+) cells under normal culture conditions. 
A
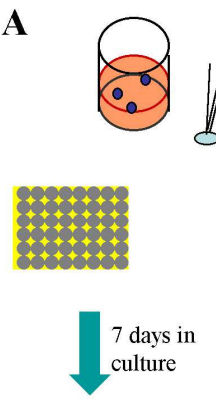

Cytochemical staining for ALP activity $(\mathrm{B}, \mathrm{C})$
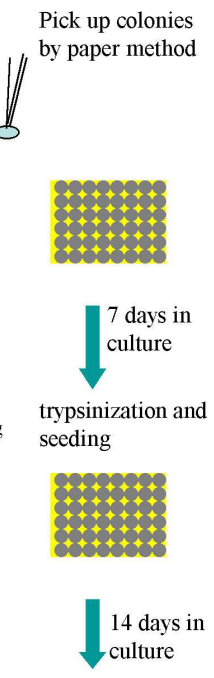

Cytochemical staining for ALP activity (D, E)
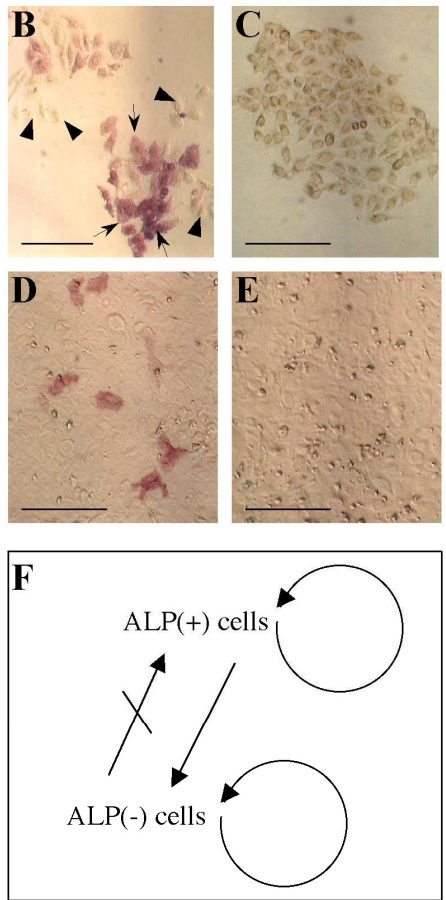

Figure 3. (A) Experimental flow for colony isolation and subsequent propagation for testing ALP activity. Colonies derived from single HeLa cells were picked using trypsin/EDTA-dipped small papers, and then transferred to the wells of a 48-well plate. Seven days after transfer of cells, the growing cells in some wells were subjected to cytochemical staining for ALP activity. Cells in the other wells were further cultured up to confluency (about 14 days after transfer of cells) and then subjected to cytochemical staining for ALP activity. (B-E) Cytochemical staining of cells for ALP activity. Bar $=100 \mu \mathrm{m}$. (F) Summary of the behavior of ALP(+) and ALP(-) HeLa cells. ALP(+) cells exhibit self-renewal and also generate ALP(-) cells. Once the ALP(-) cells generate, they continue to proliferate, as do the parental ALP(+) cells, but never revert to ALP $(+)$ cells under normal conditions.

\section{Hoechst Dye-Exclusion Assay}

CSCs are presently thought to be capable of self-renewing and simultaneously giving rise to non-CSC offspring cells with relatively differentiated properties (Al-Hajj and Clarke, 2004). In this context, our present finding that ALP (+) cells exhibit self-renewal and also generate ALP (-) cells is coincident with the above concept. However, in this case, it may be a prerequisite to know that the ALP (+) cells have the property of stemness. The ATP-binding cassette transporter G2 (ABCG2) is an efficient Hoechst 33342 efflux pump and is preferentially expressed by CSCs and somatic stem cells (Goodel et al., 1997; Jackson et al., 1999). In other words, cells with high ABCG2 activity exhibit relatively weak staining with
Hoechst 33342 dye (Goodel et al., 1997; Jackson et al., 1999). To examine the possibility that ALP (+) HeLa cells would express a high degree of ABCG2 activity, we stained colonies ( 7 days after plating of a single HeLa cell) with $5 \mathrm{mg} / \mathrm{ml}$ of Hoechst 33342 for $15 \mathrm{~min}$ at $37^{\circ} \mathrm{C}$; colonies were then fixed and subjected to cytochemical staining for ALP activity. All of the composite cells in U-type colonies exhibited uniform Hoechst 33342-derived fluorescence (Fig. 4A, B). Notably, the cells (arrows in Fig. 4A, B) exhibiting strong ALP activity also fluoresced strongly. In addition, cells comprising N-type colonies exhibited uniform Hoechst 33342derived fluorescence (Fig. 4C, D). These results suggest that ALP (+) HeLa cells do not express higher levels of ABCG2. 

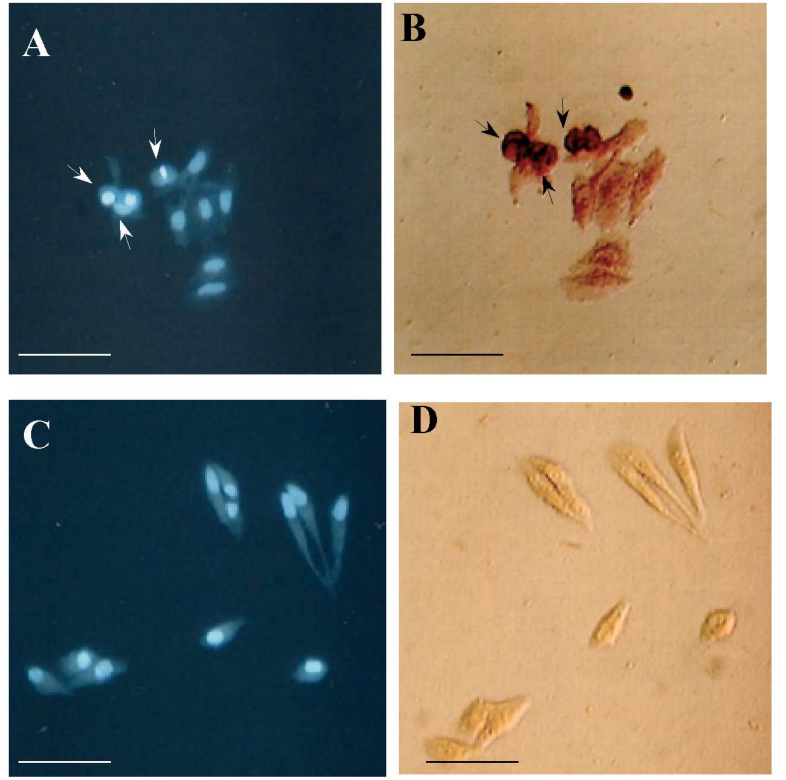

Figure 4. (A) Hoechst dye-exclusion assay. Colonies derived from single HeLa cells were first incubated in the presence of $5 \mathrm{mg} / \mathrm{ml}$ of Hoechst 33342 (A, C) for $15 \mathrm{~min}$ at $37^{\circ} \mathrm{C}$, then fixed and subjected to cytochemical staining for ALP activity (B, D). The colony classified as ' $U$ ' (B) exhibited bright Hoechst 33342 fluorescence (A). Particularly, cells exhibiting a high degree of ALP activity (arrows in A and B) exhibited distinct fluorescence. The colony classified as 'N' (D) exhibited bright fluorescence (C), similar to the U-type colony (A). Bar $=50 \mu \mathrm{m}$.

\section{Microarray Analysis}

Next, we performed microarray analysis to examine whether gene expression profiles differ between parental HeLa cells and their ALP (-) derivative $\mathrm{H}-1$ cells. Total RNA was isolated from these cells, and subjected to DNA microarray analysis using an Affymetrix kit. As shown in Fig. 5A, scatter plot analysis demonstrated a high degree of similarity in gene expression profiles between these two types of cells, but some genes exhibited different expression profiles. Of those, the 10 most strongly upregulated or downregulated genes are listed in Table 2. Interestingly, the expression of sex hormone-responsive genes, namely glycoprotein hormone $\alpha$ chain (CGA) and ras-related and estrogen-regulated growth inhibitor (RERG), was upregulated 18.2- and 9.6-fold, respectively, in the HeLa cells. Expression of ALP mRNA was also 9.2fold upregulated in these cells (see details submitted to GEO [accession number GSE42222]). Furthermore, expression of genes [Frizzled-10 (FZD10)] involved in the Wnt/ $\beta$-catenin signaling pathway was upregulated 10.5-fold in the parental HeLa cells (Table 2), suggesting the possible involvement of $\mathrm{Wnt} / \beta$-catenin signaling in the transition of ALP (+) to (-) cells. 


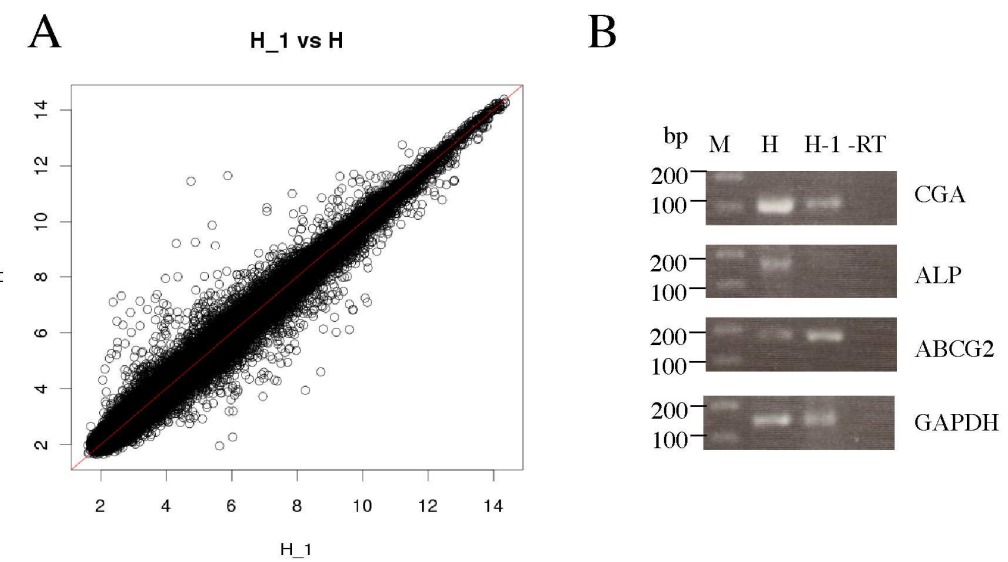

Figure 5. (A) A scatter plot of normalized signal intensities ( $\log _{2}$-transformed) between H-1 (HeLa cellderived ALP(-) cell clone) and $\mathrm{H}$ (HeLa) cells. The plots show a relatively common variability. The figure was generated using R software [http://www.r-project.org/]. (B) RT-PCR analysis of mRNA levels of CGA, ALP, ABCG2, and GAPDH in $\mathrm{H}(\mathrm{HeLa})$ and $\mathrm{H}-1$ cells. Total RNA was reverse transcribed, as described in the Materials and Methods. The resulting cDNA was subjected to PCR using the primer sets listed in Table 1. -RT, no-reverse transcription controls; M, 100-bp ladder markers from Wako Pure

Chemical Industries Inc.

\section{RT-PCR Analysis}

To confirm the altered expression levels, two selected genes (CGA and ALP, which are both upregulated in HeLa cells) were tested by semi-quantitative RT-PCR. We examined the expression levels of these two genes in the HeLa cells and the $\mathrm{H}-1$ cells. The results are shown in Fig. 5B. The expression levels of CGA and ALP in the HeLa cells were higher than those in the $\mathrm{H}-1$ cells, as expected. Expression of ABCG2 mRNA was detectable in both cell types, but its strength appeared not to differ between these two cells. These results support the fidelity of the microarray analysis performed in this study.

\section{BIO Treatment}

The above results obtained from the microarray analysis suggest the possible involvement of the Wnt/ $\beta$-catenin pathway in the transition from ALP (+) to ( -$)$ cells. In other words, when ALP (+) cells, in which the Wnt/ $\beta$-catenin pathway is probably activated, are converted to ALP (-) cells, this pathway becomes inactive, probably due to the absence of the Wnt ligands capable of binding to the cell surface receptor FZD10. $\mathrm{BIO}$, a potent synthetic inhibitor of G3SK, can activate the Wnt/ $\beta$-catenin pathway in mammals (Sato et al., 2004). Thus, it is expected that BIO treatment of ALP (+) cells would inhibit the transition from ALP ( + ) to (-) cells. To test this possibility, single HeLa cells were plated at a clonal density in wells of a 24-well plate. Seven days after seeding, the medium was changed to medium containing 1 or $3 \mu \mathrm{M} \mathrm{BIO}$ and culture was continued for up to 3 days. Cells were then fixed for cytochemical staining for ALP activity. Based on the staining pattern, as described in the Materials and Methods, colonies were classified as $\mathrm{U}, \mathrm{M}$, or $\mathrm{N}$, and the number of each type was counted. The results are shown in Fig. 6. In the treatment with $1 \mu \mathrm{M} \mathrm{BIO}$, the ratio of M-type colonies decreased, while that of U-type colonies increased. Similar results were also obtained when cells were treated with $3 \mu \mathrm{M}$ BIO. However, the ratio of $\mathrm{N}$-type colonies was not altered by the absence or presence of BIO. 


\section{Discussion}

HeLa cells are one of the most commonly used cell lines in biological research. Their immortality and capacity to grow well in vitro facilitate examination of various characteristics, including the cell cycle, perturbation, signaling pathways, and cancer metastasis. Their expression of ALP was reported by Hugon et al. (1967).

To date, most studies have focused on cellular localization of ALP (Bosmann et al., 1968; Lin et al., 1976), and regulation of ALP expression using drugs (Borgers, 1973). However, no reports have so far addressed the mosaic expression of ALP among HeLa cells, or the differences in gene expression profiles between HeLa cells [a mixture of ALP $(+)$ and $(-)$ cells] and their ALP $(-)$ derivative $\mathrm{H}-1$ cells.

As mentioned previously, ALP hydrolyzes a broad range of monophosphate esters at alkaline $\mathrm{pH}$ optima. KO mice lacking ALP function exhibited a complete defect in bone mineralization and died approximately 4 weeks after birth (Waymire et al., 1995; Narisawa et al., 1997). This abnormality resembles the human genetic defect hypophosphatasia (Narisawa et al., 1997). Deficiency of ALP expression does not affect differentiation of osteoblasts, but causes a defect in mineralization of osteoblasts (Wennberg et al., 2000). Thus, ALP is needed to maintain and support life. However, overexpression of ALP appears not to affect individual health and survival. In fact, viable and healthy transgenic mice expressing ALP have been produced, and are now recognized as a novel type of reporter mice, similar to LacZ or EGFP-expressing transgenic mice (DePrimo et al., 1996). Interestingly, ALP expression is prominent in immature, undifferentiated cells (O'Connor et al., 2008) such as ES/iPS cells, somatic stem cells, and germ cells. The reason why these cells exhibit such high levels of ALP activity remains unknown. Given that ALP expression is usually associated with stem cells, it would be reasonable to consider that ALP (+) HeLa cells may have the property of stemness. One of the prominent properties of stem cells is their strong ability to exclude Hoechst 33342 dye, which is mediated by ABCG2 protein (Zhou et al., 2001). In fact, it has been reported that ALP (+) cells isolated from the human heart have high ABCG2 activity (de Aguiar et al., 2011). Unfortunately, the present Hoechst 33342 exclusion assay revealed that the ALP (+) HeLa cells were brightly stained, as were the ALP (-) H-1 cells (see Fig. 4). We observed ABCG2 mRNA expression in both types of cells (see Fig. 5B). Taken together, expression levels of ABCG2 appear to be still low in those cells. Since other molecules, such as CD133 and CD44 high/CD24 low (heat stable antigen) are known to be markers for CSCs (Al-Hajj et al., 2003: Morel et al., 2008), it would be of interest to test whether the ALP (+) HeLa cells can express these known CSC markers.

The present DNA microarray analysis revealed that expression levels of CGA and RERG in HeLa cells, both of which encode sex hormone-responsive proteins, were higher than in their ALP (-) derivative $\mathrm{H}-1$ cells (see Table 2). These results appear to be reasonable if HeLa cells are derived from squamous cell carcinoma of the uterine cervix. In other words, the $\mathrm{H}-1$ cells may have lost one of the properties associated with their parental HeLa cells. This analysis also demonstrated that expression of some genes (including FZD10) relating to $\mathrm{Wnt} / \beta$-catenin signaling were upregulated in ALP (+) HeLa cells. Although the present study did not show the presence of other components such as E-cadherin and $\beta$-catenin, all of which are key factors in Wnt/ $\beta$-catenin signaling, there have been several reports that HeLa cells express Ecadherin and $\beta$-catenin (www.valasciences.com;

http://www.abcam.co.jp/E-Cadherinantibody-ab77287.html). Treatment of HeLa cells with BIO resulted in inhibition of transition from ALP (+) to (-) cells (see Fig. $6)$. Furthermore, it has been demonstrated that exposure to BIO increased the expression of $\beta$-catenin in HeLa cells 
(www.valasciences.com). Taken together, these data suggest that in ALP (+) cells the Wnt/ $\beta$-catenin signaling pathway has spontaneously been activated. Notably, it has been pointed out that canonical $\mathrm{Wnt} / \beta$ catenin signaling inhibits the osteogenic differentiation of MSCs (Boland et al., 2004; de Boer et al., 2004; Cho et al., 2006) and the odontoblast-like differentiation of dental pulp stem cells (Scheller et al., 2008).
Furthermore, this signaling plays a critical role in stem cell self-renewal and cancer (Reya and Clevers, 2005). Thus, it is possible that canonical Wnt $\beta$-catenin signaling may help to maintain ALP (+) HeLa cells in an undifferentiated state. Further analysis would be required to clarify the possible involvement of $\mathrm{Wnt} / \beta$-catenin signaling in maintenance of ALP (+) cells.

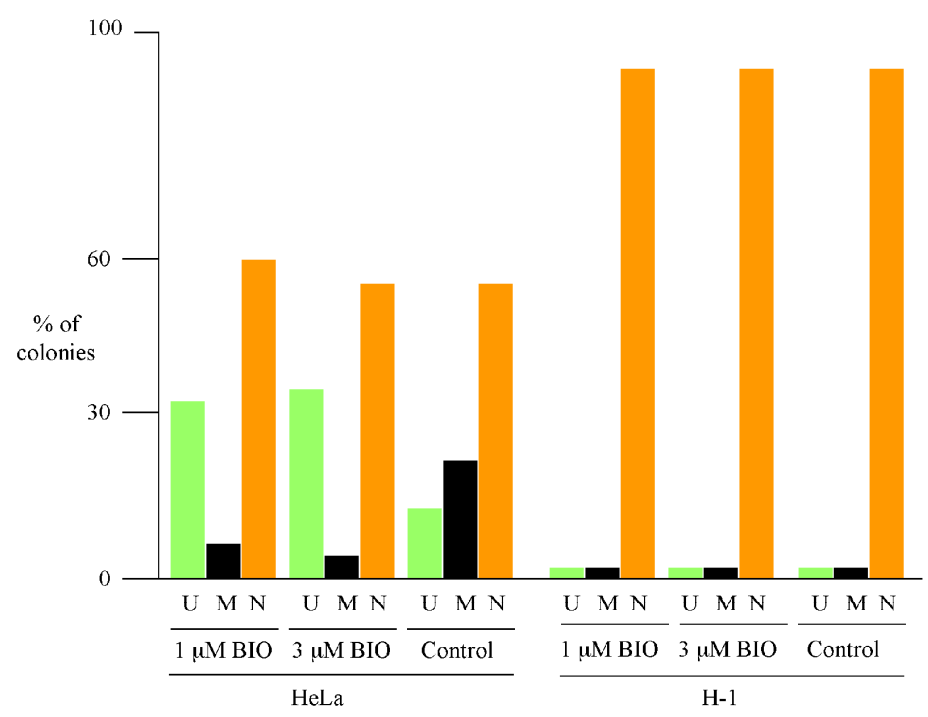

Figure 6. Effect of BIO on transition from ALP( $(+)$ to ALP(-) HeLa cells. HeLa cells or H-1 cells were plated at a clonal density into the wells of a 24-well plate, as shown in Fig. 2A. Seven days later the emerging colonies were incubated with medium containing 1 or $3 \mu \mathrm{M}$ BIO for 3 days and then subjected to cytochemical staining for AL activity using an ALP staining kit. Three $\mu \mathrm{L} / \mathrm{mL}$ of DMSO (dimethylsulfoxide; a solvent for BIO) was also added to the medium and used as a control. More than 50 colonies for each treatment were assessed for classification of the colonies, namcly U, M, and N, as previously described in Figure $2 \mathbf{C}-\mathbf{F}$. The ratio of cach type of colony in each treatment group is expressed as a percentage.

In this study, we observed that HeLa cells consist of two types of cells, namely ALP (+) and $(-)$ cells, when they are examined for cytochemical staining for ALP activity. However, it will be claimed that this phenomenon is due to contamination with other cells or to changes in cellular properties during long-term cultivation. Therefore, we tested ALP activity using HeLa cells obtained from a different laboratory. We obtained the same staining pattern (see Fig. 1B).

For more detailed analysis of the properties of ALP (+) HeLa cells, acquisition of pure populations of ALP (+) cells is a prerequisite. However, as mentioned previously, ALP (+) cells tend to convert to ALP (-) cells spontaneously. Treatment with BIO suppressed this process successfully, but its effect was only partial (see Fig. 6). Thus, it will be necessary to explore conditions that effectively block the transition from ALP (+) to ALP (-) HeLa cells. Furthermore, how to induce reversion of ALP (-) to ALP (+) cells remains unknown. If this problem can be solved, understanding of the molecular mechanism underlying transition from ALP (+) to ALP (-) HeLa cells will be accelerated. 
At present, we do not have any evidence that ALP (+) HeLa cells are CSCs. This may be in part due to the inability to isolate ALP (+) HeLa cells as a pure cell line, as mentioned above. A promising approach to overcome this problem would be the use of a construct harboring a drug resistant gene (such a neomycin resistance gene, neo) whose transcription is controlled by ALP gene promoter. If HeLa cells are transfected with this construct and subsequently subjected to selection in the presence of G418, a neomycin analog, the surviving cells would be ALP (+) cells. Untransfected cells and ALP (-) cells would have been eliminated by this treatment. To realize this hypothesis, we are now constructing such vector using a tissuenon-specific ALP promoter (Yusa et al., 2000). If these ALP (+) cells are proven to exhibit some properties characteristic of CSCs, HeLa cells would make a suitable model for CSC study.

\section{Authors' contributions}

MS and NK performed the experiments and analyzed the data. EI and IS supported the design of the experiments and the interpretation of the results. SW was responsible for the overall conception and project coordination. MO, SN, and TS provided materials used in the experiments. All authors gave final approval of the version to be published.

\section{Acknowledgments}

This work was supported by a Grant-in-Aid for Scientific Research (C) from the Ministry of Education, Science, Sports, Culture, and Technology of Japan.

\section{References}

Al-Hajj, M. \& Clarke, M. F. (2004). "SelfRenewal and Solid Tumor Stem Cells," Oncogene, 3 (43) 7274-7282.

Al-Hajj, M., Wicha, M. S., Benito-Hernandez, A., Morrison, S. J. \& Clarke, M. F. (2003). "Prospective Identification of Tumorigenic
Breast Cancer Cells," Proceedings of the National Academy of Sciences of the United States of America, 100 (7) 3983-3988.

Battula, V. L., Treml, S., Bareiss, P. M., Gieseke, F., Roelofs, H., De Zwart, P., Müller, I., Schewe, B., Skutella, T., Fibbe, W. E., Kanz, L. \& Bühring, H.- J. (2009). "Isolation of Functionally Distinct Mesenchymal Stem Cell Subsets using Antibodies Against CD56, CD271, and Mesenchymal Stem Cell Antigen1," Haematologica, 94 (2) 173-184.

Bernstine, E. G., Hooper, M. L., Grandchamp, S. \& Ephrussi, B. (1973). "Alkaline Phosphatase Activity in Mouse Teratoma," Proceedings of the National Academy of Sciences of the United States of America, 70 (12 Pt 1-2) 3899-3903.

Bièche, I., Latil, A., Parfait, B., Vidaud, D., Laurendeau, I., Lidereau, R., Cussenot, O. \& Vidaud, M. (2002). "CGA Gene (Coding for the Alpha Subunit of Glycoprotein Hormones) Overexpression in ER Alpha-Positive Prostate Tumors," European Urology, 41 (3) 335-341.

Boland, G. M., Perkins, G., Hall, D. J. \& Tuan, R. S. (2004). "Wnt 3a Promotes Proliferation and Suppresses Osteogenic Differentiation of Adult Human Mesenchymal Stem Cells," Journal of Cell Biochemistry, 93 (6) 12101230.

Bolstad, B. M., Irizarry, R. A., Astrand, M. \& Speed, T. P. (2003). "A Comparison of Normalization Methods for High Density Oligonucleotide Array Data Based on Variance and Bias," Bioinformatics, 19 (2) 185-193.

Bonnet, D. \& Dick, J. E. (1997). "Human Acute Myeloid Leukemia Is Organized as a Hierarchy That Originates from a Primitive Hematopoietic Cell," Nature Medicine, 3 (7) 730-737.

Borgers M. (1973). "The Cytochemical Application of New Potent Inhibitors of Alkaline Phosphatases," Journal of 
Histochemistry and Cytochemistry, 21 (9) 812-824.

Bosmann, H. B., Hagopian, A. \& Eylar, E. H. (1968). "Cellular Membranes: The Isolation and Characterization of the Plasma and Smooth Membranes of Hela Cells," Archives of Biochemistry and Biophysics, 128 (1) 51-69.

Chen, Z., Evans, W. H., Pflugfelder, S. C. \& Li, D.- Q. (2006). "Gap Junction Protein Connexin 43 Serves as a Negative Marker for a Stem Cell-Containing Population of Human Limbal Epithelial Cells," Stem Cells, 24 (5), 12651273.

Cho, H. H., Kim, Y. J., Kim, S. J., Kim, J. H., Bae, Y. C., Ba, B. \& Jung, J. S. (2006). "Endogenous Wnt Signaling Promotes Proliferation and Suppresses Osteogenic Differentiation in Human Adipose Derived Stromal Cells," Tissue Engineering, 12 (1) 111-121.

De Aguiar A. M., Crisciele Kuligovski, C., Da Costa, M. T. B. A., Stimamiglio, M. A., Rebelatto, C. L. K., Senegaglia, A. C., Brofman, P. R. S. B., Dallagiovanna, B., Goldenberg, S. \& Correa, A. (2011). "Alkaline PhosphatasePositive Cells Isolated from Human Hearts Have Mesenchymal Stem Cell Characteristics," Stem Cell Discovery, 1 (3) 71-80.

De Boer, J., Siddappa, R., Gaspar, C., van Apeldoorn, A., Fodde, R. \& Van Blitterswijk, C. (2004). "Wnt Signaling Inhibits Osteogenic Differentiation of Human Mesenchymal Stem Cells," Bone, 34 (5) 818-826.

Dellavalle, A., Sampaolesi, M., Tonlorenzi1, R., Tagliafico, E., Sacchetti, B., Perani, L., Innocenzi, A., Galvez, B. G., Messina, G., Morosetti, R., Li, S., Belicchi, M., Peretti, G., Chamberlain, J. S., Wright, W. E., Torrente, Y., Ferrari, S., Bianco, P. \& Cossu, G. (2007). "Pericytes of Human Skeletal Muscle Are Myogenic Precursors Distinct from Satellite Cells," Nature Cell Biology, 9 (3) 255-267.

Deprimo, S. E., Stambrook, P. J. \& Stringer, J. R. (1996). "Human Placental Alkaline
Phosphatase as a Histochemical Marker of Gene Expression in Transgenic Mice," Transgenic Research, 5 (6) 459-466.

Ginsburg, M., Snow, M. H. \& McLaren, A. (1990). "Primordial Germ Cells in the Mouse Embryo during Gastrulation," Development, 110 (2) 521-528.

Goodel, M. A., Rosenzweig, M., Kim, H., Marks, D. F., Demaria, M., Paradis, G., Grupp, S. A., $\mathrm{Sie}_{i}$, C. A., Mulligan, R. C. \& Johnson, R. P. (1997). "Dye Efflux Studies Suggest That Hematopoietic Stem Cells Expressing Low or Undetectable Levels of CD34 Antigen Exist in Multiple Species," Nature Medicine, 3 (12) 1337-1345.

Hahnel, A. C., Rappolee, D. A., Millan, J. L., Manes, T., Ziomek, C. A., Theodosiou, N. G., Werb, Z., Pedersen, R. A. \& Schultz, G. A. (1990). "Two Alkaline Phosphatase Genes Are Expressed during Early Development in the Mouse Embryo," Development, 110 (2) 555-564.

http://www.abcam.co.jp/E-Cadherinantibody-ab77287.html

Hugon, J. S., Borgers, M. \& Loni, M. C. (1967). "Alkaline Phosphatase Activity in Hela Cells," Journal of Histochemistry and Cytochemistry, 15 (4) 417-420.

Iwamura, T., Katsuki, T. \& Ide, K. (1987). "Establishment and Characterization of a Human Pancreatic Cancer Cell Line (SUIT-2) Producing Carcinoembryonic Antigen and Carbohydrate Antigen 19-9," Japanese Journal of Cancer Research, 78 (1) 54-62.

Jackson, K. A., Mi, T. \& Goodell, M. A. (1999). "Hematopoietic Potential of Stem Cells Isolated from Murine Skeletal Muscle," Proceedings of the National Academy of Sciences of the United States of America, 96 (25) 14482-14486.

Jordan, C. T., Guzman, M. L. \& Noble, M. (2006). "Cancer Stem Cells," New England Journal of Medicine, 355 (2703), 1253-1261. 
Kania, G., Corbeil, D., Fuchs, J., Tarasov, K. V., Blyszczuk, P., Huttner, W. B., Boheler, K. R. \& Wobus, A. M. (2005). "Somatic Stem Cell Marker Prominin-1/CD133 Is Expressed in Embryonic Stem Cell-Derived Progenitors," Stem Cells, 23 (6) 791-804.

Langer, D., Ikehara, Y., Takebayashi, H., Hawkes, R. \& Zimmermann, H. (2007). "The Ectonucleotidases Alkaline Phosphatase and Ntpdase2 Are Associated with Subsets Progenitor Cell Populations in the Mouse Embryonic, Postnatal and Adult Neurogenic Zones," Neuroscience, 150 (4) 863-879.

Lapidot, T., Sirard, C., Vormoor, J., Murdoch, B., Hoang, T., Caceres-Cortes, J., Minden, M., Paterson, B., Caligiuri, M. A. \& Dick, J. E. (1994). "A Cell Initiating Human Acute Myeloid Leukaemia after Transplantation into SCID Mice," Nature, 367 (6464) 645648.

Lepire, M. L. \& Ziomek, C. A. (1989). "Preimplantation Mouse Embryos Express a Heat-Stable Alkaline Phosphatase," Biology of Reproduction, 41 (3), 464-473.

Lin, C. W., Sasaki, M., Orcutt, M. L., Miyayama, H. \& Singer, R. M. (1976). "Plasma Membrane Localization of Alkaline Phosphatase in Hela Cells," Journal of Histochemistry and Cytochemistry, 24 (6) 659-667.

Morel, A.- P., Lièvre, M., Thomas, C., Hinkal, G., Ansieau, S. \& Puisieux, A. (2008). "Generation of Breast Cancer Stem Cells through Epithelial-Mesenchymal Transition," Plos ONE, 3 (8) E2888.

Mornet, E., Stura, E., Lia-Baldini, A.- S., Stigbrand, T., Ménez, A. \& Le Du, M.- H. (2001). "Structural Evidence for a Functional Role of Human Tissue Nonspecific Alkaline Phosphatase in Bone Mineralization," Journal of Biological Chemistry, 276 (33) 3117131178.

Nakayama, A., Sato, M., Shinohara, M., Matsubara, S., Yokomine, T., Akasaka, E., Yoshida, M. \& Takao, S. (2007). "Efficient
Transfection of Primarily Cultured Porcine Embryonic Fibroblasts Using the Amaxa Nucleofection System ${ }^{\mathrm{TM}}$," Cloning Stem Cells, 9 (4) 523-534.

Narisawa, S., Frohlander, N. \& Millán, J. L. (1997). "Inactivation of Two Mouse Alkaline Phosphatase Genes and Establishment of a Model of Infantile Hypophosphatasia," Developmental Dynamics, 208 (3) 432-446.

O'Connor, M. D., Kardel, M. D., Iosfina, I., Youssef, D., Lu, M., Li, M. M., Vercauteren, S., Nagy, A. \& Eaves, C. J. (2008). "Alkaline Phosphatase-Positive Colony Formation Is a Sensitive, Specific, and Quantitative Indicator of Undifferentiated Human Embryonic Stem Cells," Stem Cells, 26 (5) 1109-1116.

Quackenbush, J. (2002). "Microarray Data Normalization and Transformation," Nature Genetics, 32 (Suppl) 496-501.

Rebelatto, C. K., Aguiar, A. M., Moretão, M. P., Senegaglia, A. C., Hansen, P., Barchiki, F., Oliveira, J., Martins, J., Kuligovski, C., Mansur, F., Christofis, A., Amaral, V. F., Brofman, P. S., Goldenberg, S., Nakao, L. S. \& Correa, A. (2008). "Dissimilar Differentiation of Mesenchymal Stem Cells from Bone Marrow, Umbilical Cord Blood, and Adipose Tissue," Experimental Biology and Medicine, 233 (7) 901-913.

Reya, T. \& Clevers, H. (2005). "Wnt Signalling in Stem Cells and Cancer," Nature, 434 (7035) 843-850.

Sato, N., Meijer, L., Skaltsounis, L., Greengard, P. \& Brivanlou, A. H. (2004). "Maintenance of Pluripotency in Human and Mouse Embryonic Stem Cells through Activation of Wnt Signaling by a Pharmacological GSK-3Specific Inhibitor," Nature Medicine, 10 (1) 55-63.

Singh, S. K., Hawkins, C., Clarke, I. D., Squire, J. A., Bayani, J., Hide, T., Henkelman, R. M., Cusimano, M. D. \& Dirks, P. B. (2004). "Identification of Human Brain Tumour 
Initiating Cells," Nature, 432 (7015) 396401.

Sobiesiak, M., Sivasubramaniyan, K., Hermann, C., Tan, C., Orgel, M., Treml, S., Cerabona, F., De Zwart, P., Ochs, U., Müller, C. A., Gargett, C. E., Kalbacher, H. \& Bühring, H. J. (2009). "The Mesenchymal Stem Cell Antigen MSCA-1 Is Identical to Tissue Non-Specific Alkaline Phosphatase," Stem Cells and Development, 19 (5) 669-677.

Sutherland, A. E., Sanderson, R. D., Mayes, M., Seibert, M., Calarco, P. G., Bemfield, M. \& Damsky, C. H. (1991). "Expression of Syndecan, a Putative Low Affinity Fiboblast Growth Factor Receptor, in the Early Mouse Embryo," Development, 113 (1) 339-351.

Tomlinson, M. J., Dennis, C., Yang, X. B. \& Kirkham, J. (2011). "Tissue Non-Specific Alkaline Phosphatase Expression by Human Dental Pulp Cells: A Selectable Marker for Mineralisation," European Cells and Materials, 22 (Suppl. 3) 48.

Visvader, J. E. \& Lindeman, G. J. (2008). "Cancer Stem Cells in Solid Tumours: Accumulating Evidence and Unresolved Questions," Nature Reviews Cancer, 8 (10) 755-768.

Waymire, K. G., Mahuren, J. D., Jaje, J. M., Guilarte, T. R., Coburn, S. P. \& Macgregor, G. R. (1995). "Mice Lacking Tissue Non-Specific Alkaline Phosphatase Die from Seizures Due to Defective Metabolism of Vitamin B-6," Nature Genetics, 11 (1) 45-51.
Wennberg, C., Hessle, L., Lundberg, P., Mauro, S., Narisawa, S., Lerner, U. H. \& Millán, J. L. (2000). "Functional Characterization of Osteoblasts and Osteoclasts from Alkaline Phosphatase Knockout Mice," Journal of Bone and Mineral Research, 15 (10) 1879-1888.

Wheatley, S. C., Isacke, C. M. \& Crossley, P. H. (1993). "Restricted Expression of the Hyaluronan Receptor, CD44, During Postimplantation Mouse Embryogenesis Suggests Key Roles in Tissue Formation and Patterning," Development, 119 (2) 295-306.

www.valasciences.com; Vala Sciences Application Note: Monitoring Expression and Distribution of $\beta$-Catenin

Yusa, N., Watanabe, K., Yoshida, S., Shirafuji, N., Shimomura, S., Tani, K., Asano, S. \& Sato, N. (2000). "Transcription Factor Sp3 Activates the Liver/Bone/Kidney-Type Alkaline Phosphatase Promoter in Hematopoietic Cells," Journal of Leukocyte Biology, 68 (5) 772-777.

Zhou, S., Schuetz, J. D., Bunting, K. D., Colapietro, A. M., Sampath, J., Morris, J. J., Lagutina, I., Grosveld, G. C., Osawa, M., Nakauchi, H. \& Sorrentino, B. P. (2001). "The ABC Transporter Bcrp1/ABCG2 Is Expressed in a Wide Variety of Stem Cells and Is A Molecular Determinant of the SidePopulation Phenotype," Nature Medicine, 7 (9) 1028-1034. 